\title{
Associations between residual depressive symptoms, cognition, and functioning in patients with euthymic bipolar disorder: results from the FACE-BD cohort
}

Paul Roux, Aurélie Raust, Anne-Sophie Cannavo, Valérie Aubin, Bruno Aouizerate, Jean-Michel Azorin, Frank Bellivier, Raoul Belzeaux, Thierry Bougerol, Iréna Cussac, Philippe Courtet, Bruno Etain, Sébastien Gard, Sophie Job, Jean-Pierre Kahn, Marion Leboyer, Emilie Olié, the FondaMental Advanced Centers of Expertise in Bipolar Disorders (FACE-BD) Collaborators, * Chantal Henry and Christine Passerieux

\section{Background}

The relationship between residual depressive symptoms, cognition and functioning in patients with euthymic bipolar disorder is a subject of debate.

\section{Aims}

To assess whether cognition mediates the association between residual depressive symptoms and functioning in patients with bipolar disorder who were euthymic.

\section{Method}

We included 241 adults with euthymic bipolar disorder in a multicentre cross-sectional study. We used a battery of tests to assess six cognition domains. A path analysis was then used to perform a mediation analysis of the relationship between residual depressive symptoms, cognitive components and functioning.

\section{Results}

Only verbal and working memory were significantly associated with better functioning. Residual depressive symptoms were associated with poorer functioning. No significant relationship was found between residual depressive symptoms and any cognitive component.

\section{Conclusions}

Cognition and residual depressive symptoms appear to be two independent sources of variation in the functioning of people with euthymic bipolar disorder.

\section{Declaration of interest}

None.

\section{Copyright and usage}

(c) The Royal College of Psychiatrists 2017.
Bipolar disorders are highly disabling ${ }^{1}$ and prevalent. ${ }^{2}$ More than half of individuals with bipolar disorder experience significant functional impairment in several domains, such as family and social life and work, outside the acute phases of the illness. ${ }^{3}$ In the past decade, the focus of research in bipolar disorder has changed from clinical remission to functional recovery. ${ }^{3}$ However, the sources of high variation observed in the psychosocial functioning of individuals with bipolar disorder are still poorly understood. Cognitive impairment is an important determinant of functional impairment in bipolar disorder. ${ }^{4}$ Because the strength of association between poor social functioning and cognitive impairment in bipolar disorder is similar to that seen in schizophrenia, ${ }^{5}$ it seems crucial to characterise the underlying architecture of cognitive performance in bipolar disorder. Previous studies investigating multiple cognitive areas in bipolar disorder have usually focused on domains a priori that were subjectively defined and selected. However, the validity of these domains is questionable, as it relies on the assumption that the latent organisation of human cognition is similar in people with bipolar disorder and healthy controls. This crucial assumption has received little experimental evidence. ${ }^{6}$ It thus remains possible that the same neuropsychological tests might evaluate different cognitive competencies in these two groups of participants if there is a discrepancy in how cognitive measures relate to one another between individuals with and without bipolar disorder. This lack of equivalence between the constructs could lead to artefacts in the observed differences in cognitive test performances. A few

*See the Appendix for the list of collaborators. studies have explored the latent cognitive structure in bipolar disorder and found that cognitive functioning was best described by multifactorial models. ${ }^{6-8}$ However, some of these studies included patients who were symptomatic. ${ }^{7,8}$ Here, we have measured performance for a broad range of cognitive domains in euthymic bipolar disorder using a comprehensive battery of neuropsychological tests. We have examined the component structure of bipolar disorder cognitive processes using a principal component analysis (PCA), which allows the data-driven reduction of multiple cognitive measures, avoids arbitrary and a priori categorisation of several tests into domains, and results in a reliable estimate of underlying cognitive constructs in bipolar disorder.

Among clinical factors, residual depressive symptoms have been reported to be the strongest predictor of functional impairment ${ }^{9}$ and quality of life ${ }^{10}$ in euthymic bipolar disorder. They are also associated with lower adherence to medication in individuals with bipolar disorder. ${ }^{11}$ In contrast, residual hypomanic symptoms have no impact on functioning in euthymic bipolar disorder. ${ }^{4,9,12-14}$ Whereas residual hypomanic symptoms are not related to cognition, ${ }^{15}$ it is less clear whether subthreshold depressive symptoms negatively affect neuropsychological performance in euthymic bipolar disorder or not. The relationship between residual depressive symptoms and cognition is mixed, showing a small impact of subthreshold depression on only a few cognitive components, such as verbal memory, speed, and executive function, but not for others. ${ }^{16} \mathrm{~A}$ few studies have explored the role of cognition in mediating the relationship between depressive symptoms and functioning in euthymic 
bipolar disorder, with inconsistent results. One study showed that verbal memory partially mediated the relationship between subthreshold depressive symptoms and functional outcome in people with bipolar disorder who were euthymic. ${ }^{17}$ However, the sample size was not large enough to include other cognitive moderators in the model. In contrast, another study reported that cognition did not mediate the relationship between depressive symptoms and social competencies in bipolar disorder. ${ }^{18}$ Again, only one global neurocognitive composite score was included as the cognitive mediator in the model, because of the limited sample size. There have been no studies, to date, that have investigated simultaneous mediation between subdepressive symptoms and functioning by multiple cognitive components in bipolar disorder. In this study, the cognitive domains obtained with PCA were entered in a path analysis model, as potential mediators between residual depressive symptoms and functioning.

\section{Method}

\section{Study design and recruiting network characteristics}

This multicentre, cross-sectional study included patients recruited into the FACE-BD (FondaMental Academic Centers of Expertise for Bipolar Disorders) cohort by a French national network of nine bipolar disorder expert centres (Bordeaux, Créteil, Grenoble, Marseille, Monaco, Montpellier, Nancy, Paris and Versailles). This network was set up by the FondaMental Foundation (www.fondation-fondamental.org) and funded by the French Ministry of Research and the French Ministry of Health to build an infrastructure and provide resources to follow clinical cohorts and comparative-effectiveness research on a representative patient population.

\section{Participants}

Bipolar disorder was diagnosed based on a structured clinical interview that assessed the DSM-IV-TR criteria. ${ }^{19}$ Out-patients of 18 to 65 years of age with type I, II, or not otherwise specified (NOS, including cyclothymia) bipolar disorder were eligible. All patients were euthymic when they were tested according to the DSM-IV-TR criteria, with a Montgomery-Åsberg Depression Rating Scale (MADRS) ${ }^{20}$ of less than 10 and a Young Mania Rating Scale (YMRS) ${ }^{21}$ of less than 10. Exclusion criteria were a history of neurological or sensory disorders, dyslexia, dysorthographia, dyscalculia, dysphasia, dyspraxia, language delay, substance-related disorders in the previous month and electroconvulsive therapy in the past year. The ethics committee (Comite de Protection des Personnes Ile de France IX) approved the study protocol on 18 January 2010. Although the committee waived the requirement for written informed consent, the patients received a letter informing them of the study and asking whether they agreed to participate.

\section{Assessment tools}

\section{Clinical assessments}

The age at onset; number of previous mixed, hypomanic, manic and major depressive episodes; subtype of bipolar disorder; and history of psychotic symptoms were recorded. We used the yes/ no format for recording whether the patient was taking lithium carbonate, anticonvulsants, antipsychotics, antidepressants or anxiolytics at the time of the evaluation. Finally, three sociodemographic characteristics were collected: gender, age and educational level.

Psychosocial functioning was measured using the Functioning Assessment Short Test (FAST), ${ }^{22}$ which covers six functioning domains: autonomy, occupational functioning, cognitive functioning, financial issues, interpersonal relationships and leisure time. The higher the score, the greater the disability. FAST has good internal consistency (Cronbach alpha between 0.87 and $0.96)$ and test-retest reliability (Pearson correlation coefficient between 0.90 and 0.97 ; intraclass correlation coefficient between 0.90 and 0.98 , see online supplement DS1 for a bibliography of FAST psychometric properties).

\section{Battery of cognitive tests}

Experienced psychologists administered the tests in a fixed order. Testing lasted a total of $120 \mathrm{~min}$, including a 5-10 min break. The standardised test battery complied with the recommendations issued by the International Society for Bipolar Disorders. ${ }^{23}$ It included 11 tests and evaluated the following six cognitive domains:

(a) motor speed with the digit symbol coding and symbol search subtests from the Wechsler Adult Intelligence Scale (WAIS) version III; ${ }^{24}$

(b) attention with the Conners' Continuous Performance Test II $(\mathrm{CPT}-\mathrm{II})^{25}$ and the Trail Making Test (TMT); ${ }^{26}$

(c) executive functions with the Stroop colour and word test and verbal fluency; ${ }^{27}$

(d) verbal memory with the California Verbal Learning Test (CVLT) $;^{28}$

(e) working memory with the digit span subtest from the WAIS-III and the spatial span subtest from the Wechsler Memory Scale version III; and

(f) intellectual functioning with the vocabulary and matrix reasoning subtests from the WAIS-III.

Some of the current cognitive data obtained with this battery have been published previously. ${ }^{29}$

\section{Statistical analyses}

PCA

PCA is a powerful statistical method for identifying the underlying organisation of multiple variables such as cognitive measures and allows data reduction necessary to avoid type I errors from multiple comparisons. The data-set for the PCA included 22 raw cognitive variables: number of correct answers for the digit symbol coding and symbol search tests, percentages of omissions and commission errors for the CPT-II, reaction time (ms) for hits in the CPT, time (s) to complete the TMT-A and -B, number of responses in the colour, word, and colour-word conditions of the Stroop test, number of correct words for phonemic and semantic verbal fluency, number of recalled words in the immediate recall, short and long delay free recall of the CVLT, number of total correct recognised words for the CVLT, span lengths for the forward and backward conditions of the spatial and digit span tests, number of correct answers for matrix reasoning, and total score for vocabulary.

Sampling adequacy was evaluated using the Kaiser-MeyerOlkin (KMO) measure ${ }^{30}$ for the overall cognitive data-set and Bartlett's test of sphericity. ${ }^{31}$ The number of components to be extracted in the PCA was determined by Horn's parallel analysis. ${ }^{32}$ This method contrasts eigenvalues produced through a parallel PCA on 1000 random data-sets, with the same number of variables and observations as the observational data-set, to generate eigenvalues for components that are adjusted for sample error-induced inflation. Adjusted eigenvalues $>1$ indicate dimensions to retain.

We ran a PCA on cognitive variables followed by an Oblimin rotation. The rotation was performed to simplify the component 
structure. We used an oblique rotation because the cognitive components were believed to be correlated with each other. In PCA, the usual standard for sample size is a participant-to-variable ratio $>5,{ }^{33}$ and therefore required 110 participants for the current study.

\section{Path and mediation analyses}

Zero-order correlations between MADRS, cognitive components, FAST scores, age, gender and education were calculated using Pearson correlation coefficients. A path analysis was performed using MADRS, cognitive components and FAST scores to test whether cognitive components mediated the relationship between residual depressive symptoms and functioning. The model tested in the path analysis did not include the YRMS score, as we expected it to correlate with neither cognition nor functioning. The model allowed the residual variances of the cognitive components scores to be correlated. Age, gender and education were used as covariates in the model.

Analyses were performed using the lavaan package of $R$ statistical software version 3.3 with the maximum likelihood estimation method. Linear regression analyses were conducted to evaluate the relationships among the variables and were indexed using standardised path coefficients. Because the FAST total score is usually not normally distributed in euthymic bipolar disorder, ${ }^{34}$ we used a non-parametric bootstrapping of the standard errors with 2000 iterations for the correlation and path analyses. The fit between the model and the data was assessed using four indices: the chi-square goodness-of-fit statistic $\left(\chi^{2}\right)$, comparative fit index (CFI), root-mean-squared error of approximation (RMSEA) and standardised root-mean-square residual (SRMR).

\section{Results}

\section{Participants}

We included 241 patients. Table 1 reports their sociodemographic and clinical characteristics and Table 2 the results of the battery of

\begin{tabular}{|c|c|}
\hline Variable & \\
\hline Age, years: mean (s.d.) range & $41(11.3)$ 19-65 \\
\hline Educational level, years: mean (s.d.) range & $14.5(2.7) 7-22$ \\
\hline Age at onset, years: mean (s.d.) range & $25(9.5) 12-60$ \\
\hline $\begin{array}{l}\text { Number of episodes, mean (s.d.) range } \\
\text { Mixed } \\
\text { Hypomanic } \\
\text { Manic } \\
\text { Major depressive }\end{array}$ & $\begin{array}{l}0.2(0.7) 0-8 \\
2.8(5.3) 0-30 \\
1.4(1.9) 0-10 \\
5.2(5.4) 0-30\end{array}$ \\
\hline $\begin{array}{l}\text { Scores, mean (s.d.) range } \\
\text { Montgomery-Äsberg Depression Rating Scale (0-60) } \\
\text { Young Mania Rating Scale (0-60) } \\
\text { Functioning Assessment Short Test }(0-72)\end{array}$ & $\begin{array}{c}4(3.4) 0-10 \\
1.1(2) 0-10 \\
16.8(13.2) 0-64\end{array}$ \\
\hline Gender, \% male & 40.2 \\
\hline $\begin{array}{l}\text { Diagnosis, \% } \\
\text { Type I } \\
\text { Type II } \\
\text { Not otherwise specified }\end{array}$ & $\begin{array}{l}55.6 \\
29.5 \\
14.9 \\
\end{array}$ \\
\hline History of psychosis, \% & 43.6 \\
\hline $\begin{array}{l}\text { Medication, \% } \\
\text { Antidepressant } \\
\text { Lithium carbonate } \\
\text { Anticonvulsant } \\
\text { Antipsychotic }\end{array}$ & $\begin{array}{l}26 \\
25 \\
32.7 \\
28.4\end{array}$ \\
\hline
\end{tabular}

cognitive tests. No patient had more than $5 \%$ missing cognitive data; the missing cognitive data were estimated using a multivariate imputation by chained equations in the mice package of $R$.

\section{PCA}

The KMO measure for the overall cognitive data-set was 0.85 and Bartlett's test of sphericity was significant $\left(\chi^{2}(231)=2295, P<0.001\right)$, both indicating good factorability of the cognitive data. Horn's parallel analysis showed that five components should be retained, as their adjusted eigenvalue was above 1 (online Table DS1). The five-component structure explained $61 \%$ of the variance (online Table DS2). All component loadings were greater than 0.4 , and all communalities were higher than 0.3 (Table 3 ). The first component consisted of all measures of the CVLT and was designated 'verbal memory'. The second component bundled TMT, CPT omissions, symbol search, vocabulary, symbol coding and semantic verbal fluency and was designated 'speed of processing and verbal knowledge'. The third component included all measures of spatial and digit spans, with matrix reasoning, and was designated 'working memory and problem-solving'. The fourth component consisted of all measures of the Stroop test, with phonemic verbal fluency, and was designated 'verbal fluency and inhibition'. The final component included CPT reaction time and commission and was designated 'visual sustained attention'.

\section{Table 2 Participant neuropsychological performance}

\begin{tabular}{|c|c|c|}
\hline Variable & $\begin{array}{l}\text { Percentile, } \\
\text { mean (s.d.) }\end{array}$ & $\begin{array}{l}\text { Raw, mean } \\
\text { (s.d.) }\end{array}$ \\
\hline \multicolumn{3}{|l|}{ Motor speed } \\
\hline Digit/symbol coding & $43.1(25.8)$ & $70.2(15.2)$ \\
\hline Symbol search & $56.2(28)$ & $33.7(7.5)$ \\
\hline \multicolumn{3}{|l|}{ Attention } \\
\hline \multicolumn{3}{|c|}{ Continuous Performance Test } \\
\hline Omissions $^{a}$ & $43.1(27.9)$ & $2.2(4.6)$ \\
\hline Commissions $^{a}$ & $53.6(30.1)$ & $30.8(20.2)$ \\
\hline Reaction time ${ }^{b}$ & $52(32.6)$ & $402.5(84.2)$ \\
\hline \multicolumn{3}{|l|}{ Trail Making Test ${ }^{c}$} \\
\hline Part A & $49.6(25.7)$ & $35(13)$ \\
\hline Part B & $45.5(28.3)$ & $104.1(15.9)$ \\
\hline \multicolumn{3}{|l|}{ Executive functions } \\
\hline \multicolumn{3}{|l|}{ Stroop test } \\
\hline Word & $45.8(22.8)$ & $104.1(15.9)$ \\
\hline Colour & $37.4(25.2)$ & $73(12.9)$ \\
\hline Colour/word & $47(29.8)$ & $43.1(11.1)$ \\
\hline \multicolumn{3}{|l|}{ Verbal fluency } \\
\hline Phonemic & $48.2(31.3)$ & $24.1(7.3)$ \\
\hline Semantic & $39.9(27.9)$ & $31.7(8)$ \\
\hline \multicolumn{3}{|l|}{ verbal memory } \\
\hline \multicolumn{3}{|c|}{ California Verbal Learning Test } \\
\hline Immediate recall & $46.8(32.5)$ & $56.4(9.9)$ \\
\hline Short delay free recall & $46.9(30)$ & $11.9(2.9)$ \\
\hline Long delay free recall & $44.8(30.3)$ & $12.2(2.7)$ \\
\hline Total recognition & $52.8(26.3)$ & $15.1(1.2)$ \\
\hline \multicolumn{3}{|l|}{ Working memory } \\
\hline \multicolumn{3}{|l|}{ Spatial span } \\
\hline Forward & $46.1(26.5)$ & $8.3(1.9)$ \\
\hline Backward & $43.9(26)$ & $7.4(1.8)$ \\
\hline \multicolumn{3}{|l|}{ Digit span } \\
\hline Total & $43.6(25.9)$ & \\
\hline Forward & & $9.4(2)$ \\
\hline Backward & & $6.4(2.3)$ \\
\hline \multicolumn{3}{|l|}{ Intellectual functioning } \\
\hline Matrix reasoning & $45.8(29)$ & $18.3(4.5)$ \\
\hline Vocabulary & $61.9(28)$ & $42.9(10.2)$ \\
\hline $\begin{array}{l}\text { a. Raw scores are percentage } \\
\text { b. Raw scores are in ms. } \\
\text { c. Raw scores are in s. }\end{array}$ & & \\
\hline
\end{tabular}




\begin{tabular}{|c|c|c|c|c|c|c|}
\hline \multirow[b]{2}{*}{ Variable } & \multicolumn{5}{|c|}{ Component loadings } & \multirow[b]{2}{*}{ Communality } \\
\hline & 1 & 2 & 3 & 4 & 5 & \\
\hline CVLT, long delay free recall & 0.94 & 0.03 & -0.06 & 0.04 & 0.02 & 0.88 \\
\hline CVLT, short delay free recall & 0.92 & 0.03 & -0.03 & 0.01 & 0.01 & 0.85 \\
\hline CVLT, immediate recall & 0.84 & 0.02 & 0.05 & 0.09 & -0.05 & 0.78 \\
\hline CVLT, total recognition & 0.73 & -0.15 & 0.06 & -0.23 & 0.07 & 0.55 \\
\hline TMT, B & -0.13 & -0.69 & -0.16 & 0.03 & -0.08 & 0.66 \\
\hline CPT, omissions & 0.26 & -0.65 & 0.17 & 0.04 & 0.02 & 0.37 \\
\hline Symbol search & 0.10 & 0.65 & 0.06 & 0.17 & -0.10 & 0.62 \\
\hline Vocabulary & -0.03 & 0.62 & 0.04 & 0 & 0.25 & 0.44 \\
\hline Symbol coding & 0.17 & 0.61 & 0.05 & 0.21 & -0.15 & 0.65 \\
\hline TMT A & -0.07 & -0.52 & -0.20 & -0.03 & 0.16 & 0.46 \\
\hline Verbal fluency semantic & 0.03 & 0.46 & 0.13 & 0.24 & 0 & 0.41 \\
\hline Spatial span backward & -0.02 & 0.16 & 0.74 & -0.09 & -0.03 & 0.61 \\
\hline Spatial span forward & 0.01 & 0.04 & 0.73 & -0.02 & -0.06 & 0.55 \\
\hline Digit span backward & 0.08 & -0.04 & 0.69 & 0.21 & 0.06 & 0.62 \\
\hline Digit span forward & -0.08 & -0.14 & 0.65 & 0.35 & 0.17 & 0.57 \\
\hline Matrix reasoning & 0.18 & 0.30 & 0.56 & -0.35 & 0.01 & 0.62 \\
\hline Stroop, word & -0.04 & 0.08 & 0.01 & 0.77 & 0.02 & 0.63 \\
\hline Stroop, colour & 0.13 & 0.08 & 0.10 & 0.69 & -0.06 & 0.62 \\
\hline Stroop, word/colour & 0.11 & 0.09 & 0.18 & 0.56 & -0.16 & 0.50 \\
\hline Verbal fluency phonemic & 0.09 & 0.27 & -0.18 & 0.41 & 0.34 & 0.39 \\
\hline CPT, reaction time & -0.03 & -0.13 & 0 & -0.09 & 0.86 & 0.78 \\
\hline CPT, commissions & -0.07 & -0.14 & -0.05 & -0.06 & -0.83 & 0.74 \\
\hline
\end{tabular}

\section{Path and mediation analyses}

Online Table DS3 reports the zero-order correlations between the variables included in the model. The path analysis model allowed correlations between the residual variances of all cognitive components, except 'visual sustained attention', which was not correlated with any other cognitive components.

The path analysis model is shown in Fig. 1. We represent neither covariances between cognitive components, nor the regressions on covariates, to enhance readability. Path analysis requires at least 15 participants for each variable. ${ }^{35}$ We included ten variables in the model and therefore required at least 150 participants. There were $0.8 \%$ missing data, which were handled using the full information maximum likelihood estimation. The four patterns of missingness are reported in online Table DS4 and the covariance coverage matrix of missing data in online Table DS5.

The model provided a good fit for the data, as suggested by the non-significant chi-square goodness-of-fit statistic $\left(\chi^{2}(4)=7.5\right.$, $P=0.113$ ), a CFI of 0.989 (thus greater than 0.95), an RMSEA of 0.06 (which was not significantly greater than 0.05 , as the $P$-value of the one-sided test of the null hypothesis that RMSEA was greater than 0.05 was equal to 0.329), and SRMR of 0.017 (thus lower than 0.08).

The model explained $30 \%$ of the variance in functioning. Altogether, the analysis revealed the following relationships between the variables (Fig. 1): a significant positive association between MADRS and FAST, a significant negative association between 'verbal memory' cognitive component and FAST, and a significant negative association between the 'verbal fluency and inhibition' cognitive component and FAST. We found no other significant associations, and in particular, MADRS was not significantly associated with any cognitive component, thus showing that cognitive component scores did not mediate the relationship between MADRS and FAST. Estimated standardised path coefficients for all variables included in the path analysis model (with the covariates) and residual correlation coefficients between cognitive components are reported in online Table DS6.

\section{Discussion}

\section{Main findings and comparison with other studies}

Here, we first used a PCA to identify the underlying architecture of cognitive processing in patients with bipolar disorder who were euthymic. We then used a path analysis to evaluate whether cognition mediated the relationship between residual depressive symptoms and functioning. We found five underlying components involved in cognition in euthymic bipolar disorder. Two components were derived from individual variables that were relatively homogeneous and specific regarding modality: the 'verbal memory' component was derived only from CVLT measures and the 'visual sustained attention' only from CPT measures. The remaining three components were more heterogeneous. The 'verbal fluency and inhibition' component consisted of verbal responses sometimes involving inhibition (phonemic verbal fluency and colour-word condition of the Stroop test) and sometimes not (colour and word condition of the Stroop test). The 'speed of processing and verbal knowledge' component contained a combination of visuospatial and verbal variables. The 'working memory and problem-solving' component bundled non-verbal reasoning and working memory measures. Greater variability within cognitive components has already been reported in bipolar disorder and was interpreted as a decrease in the differentiation of previously discrete cognitive processes through a decline in neural connectivity. ${ }^{8}$ The number of extracted cognitive components is similar to that of previous studies that found five ${ }^{6}$ or six underlying dimensions in cognition for bipolar 
Cognitive component scores

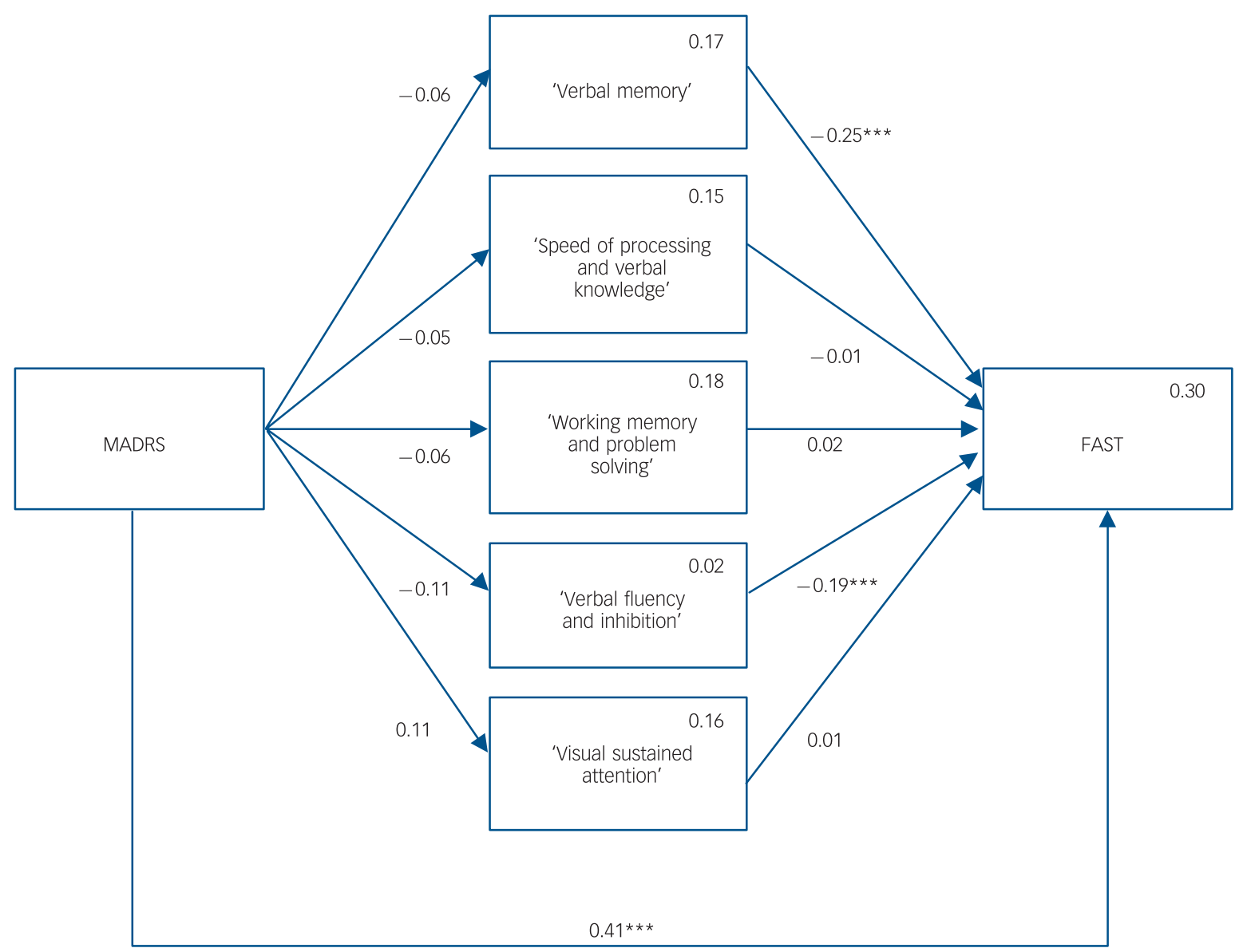

\section{Fig. 1 Mediation model.}

Rectangles represent the observed measured variables. Arrows showing the free regression weight are drawn between variables. Values are the standardised path coefficients The squared multiple correlation $(R)$ value for the dependent variable appears in the upper right corner of each rectangle. Covariates and covariation between the cognitive components are not drawn to increase readability but were included in the model. MADRS, Montgomery-Äsberg Depression Rating Scale; FAST, Functioning Assessment Short Test. ${ }^{* * *} P>0.001$.

disorder. ${ }^{7}$ The labels used in these previous studies to describe the cognitive dimensions were similar to those applied in the current study, consisting of verbal memory, speed of processing, working memory, executive functions, verbal knowledge and attention dimensions. This suggests a relative stability and reliability of the method we used to uncover the underlying cognitive components in bipolar disorder.

There was a negative association between residual depressive symptoms and functioning: individuals with more pronounced depressive symptoms had poorer social functioning, which was not explained by age, gender, lower education or poorer cognition. This finding is in accordance with previous crosssectional $^{13,17,18,34}$ and longitudinal studies, ${ }^{9,14}$ showing that subclinical depressive symptoms in bipolar disorders are the main predictors of poor functional outcome, particularly work functioning. ${ }^{36}$ The present study included only patients who were euthymic, based on stringent criteria: the mean score for depression was very low but similar to previous studies exploring social functioning in euthymic bipolar disorder. ${ }^{9,13,17,34}$ However, the data show that functional impairment may be associated with even very low residual depressive symptoms, measured with a scale that was not specifically designed to assess subsyndromal depressive symptoms.

Among the five cognitive components found in this study, only two were positively associated with functioning: 'verbal memory' and 'verbal fluency and inhibition'. Patients with better verbal memory, verbal fluency and inhibition also had a better social functioning. These results are consistent with several prior reports indicating that verbal memory ${ }^{14}$ and inhibitory control (Stroop colour-word test) ${ }^{37}$ were more highly associated with functioning than other cognitive functions. That functioning was more highly related to residual depressive symptoms than cognition could be explained by the auto-evaluation method we used to measure everyday functioning. Self-reported measures of social functioning may be influenced less by objective cognitive performance and more by depressive symptomatology, because of pessimistic subjective appraisal of oneself and one's environment. This influence might be particularly important for low FAST scores (better functioning), like in our sample, where the distribution of FAST scores was skewed on the right. In contrast, the reverse may be true for performance-based measures of functioning and real-world functional milestones in bipolar disorder, which may be influenced more by objective cognitive performance and less by depression. ${ }^{5,12}$

No associations between residual depressive symptoms and cognitive components were significant, showing that cognition does not mediate the relationship between subclinical residual depressive symptoms and functioning. This result is in accordance with a previous study reporting that perceived cognitive impairment and subclinical residual depressive symptoms are 
two independent sources of variation in the functioning of individuals with bipolar disorder. ${ }^{38}$ However, this mediation might occur for higher levels of depressive symptoms, as the impact of depressive symptoms on cognition varied according to the clinical response after treatment. ${ }^{39}$

Our model explained only $30 \%$ of the variance in functioning, supporting a role for other factors that were not measured here. Previous studies have suggested that functioning may be impaired when sleep is persistently disrupted, ${ }^{40}$ when social cognition is impaired, ${ }^{13}$ and when episode density is high. ${ }^{37}$ The mean level of functioning in participants recruited in this study corresponds to moderate functional difficulties. ${ }^{3}$ It is in the range (from 6 to 29) of those found in studies exploring the relationship between cognition and functioning in euthymic bipolar disorder. ${ }^{13,34,38}$ This consistency supports the general applicability of our findings to patients with euthymic bipolar disorder, provided the same assessment tools are used.

\section{Limitations}

Limitations of our study include the cross-sectional design, which precludes the assessment of causality and the direction of potential causal links. Another limitation is the lack of inclusion of social cognitive tasks in the assessment. We did not compare the cognitive architecture found in participants with bipolar disorder with a control group. Finally, the sample size was not large enough to test a more complex model that includes other variables of the illness (type of bipolar disorder, number of previous episodes, age at onset and history of psychosis) and medication. The time since the last mood episode might also be an important factor lacking in the present study. These variables might have also had an effect on functioning in euthymic bipolar disorder.

\section{Implications}

Our findings have important implications for future clinical studies. Individuals with bipolar disorder who respond to treatment may nevertheless continue to experience residual depressive and cognitive symptoms, leading to difficulties in functioning. First, it seems crucial to improving the assessment and the characterisation of residual depressive symptoms, with, for example, specific scales. Treatment approaches should possibly include cognitive performance improvement and full residual depressive symptom remission as important targets to obtain functional recovery in bipolar disorder. The optimal method to treat residual depressive symptoms is not clear, but they may be targeted by the use of mood stabilisers effective in the treatment of depressive polarity. Evidence for the efficacy of pharmacological and psychological interventions that target cognitive deficits in bipolar disorder is still preliminary, despite promising avenues such as functional remediation. ${ }^{41}$ Among cognitive dimensions for cognitive remediation in euthymic bipolar disorder, our data suggest that verbal memory and fluency, and inhibition may be choice targets.

In summary, this study provides further evidence that cognitive impairments in specific dimensions are a core feature of bipolar disorder. This study also suggests that cognition is a separable dimension from depressive symptoms that persist during the inter-episodic period of bipolar disorder. Verbal memory and fluency and Stroop test performance were particularly associated with functioning in our participants with euthymic bipolar disorder and should be assessed in future studies focusing on functional outcome in bipolar disorder.
Paul Roux, MD, PhD, Service Universitaire de Psychiatrie d'Adultes, Centre Hospitalier de Versailles, Le Chesnay, Laboratoire HandiRESP, EA4047, UFR des Sciences de la Santé Simone Veil, Université de Versailles Saint-Quentin-En-Yvelines, Montigny-le-Bretonneux and Fondation Fondamental, Créteil, France; Aurélie Raust, MSC, Fondation Fondamental, Créteil, AP-HP, Hôpitaux Universitaires Henri Mondor, DHU Pepsy, Pôle de Psychiatrie et d'Addictologie, Créteil, Université Paris Est, Faculté de Médecine, Créteil and Inserm, U955, Equipe Psychiatrie Translationnelle, Créteil, France; Anne-Sophie Cannavo, MSc, Service Universitaire de Psychiatrie d'Adultes, Centre Hospitalier de Versailles, Le Chesnay, Laboratoire HandiRESP, EA4047, UFR des Sciences de la Santé Simone Veil, Université de Versailles Saint-QuentinEn-Yvelines, Montigny-le-Bretonneux and Fondation Fondamental, Créteil, France; Valérie Aubin. MD, PhD, Fondation Fondamental, Créteil and Pôle de Psychiatrie, Centre Hospitalier Princesse Grace, Monaco, France; Bruno Aouizerate, MD, PhD, Fondation Fondamental, Créteil and Centre Expert Trouble Bipolaire, Pôle de Psychiatrie Générale et Universitaire (3/4/7), Hôpital Charles Perrens, Bordeaux; JeanMichel Azorin, MD, PhD, Fondation Fondamental, Créteil and AP-HM, Hôpital SainteMarguerite, Pôle de Psychiatrie, Marseille, France; Frank Bellivier, MD, PhD, Fondation Fondamental, Créteil, AP-HP, GH Saint-Louis - Lariboisière - Fernand Widal, Pôle Neurosciences, Paris and Université Paris Diderot, UMR-S, Paris; Raoul Belzeaux, MD, PhD, Fondation Fondamental, Créteil and AP-HM, Hôpital Sainte-

Marguerite, Pôle de Psychiatrie, Marseille, France; Thierry Bougerol, MD, Fondation Fondamental, Créteil, University Grenoble Alpes, Grenoble, CHU de Grenoble et des Alpes, Grenoble and Grenoble Institut des Neurosciences (GIN) Inserm U 836, La Tronche, France; Iréna Cussac, MD, Fondation Fondamental, Créteil and Pôle de Psychiatrie, Centre Hospitalier Princesse Grace, Monaco, France; Philippe Courtet $\mathrm{MD}, \mathrm{PhD}$, Fondation Fondamental, Créteil, Department of Emergency Psychiatry \& Post Acute Care, Academic hospital of Montpellier, Montpellier University, Montpellie and 16 INSERM U1061, Montpellier, France; Bruno Etain, MD, PhD, Fondation Fondamental, Créteil, AP-HP, GH Saint-Louis - Lariboisière - Fernand Widal, Pôle Neurosciences, Paris, 11 Université Paris Diderot, UMR-S, Paris, France and Centre for Affective Disorders, Department of Psychological Medicine, Institute of Psychiatry, Psychology \& Neuroscience, King's College London, London, UK; Sébastien Gard $M D$, Fondation Fondamental, Créteil and Centre Expert Trouble Bipolaire, Pôle de Psychiatrie Générale et Universitaire (3/4/7), Hôpital Charles Perrens, Bordeaux; Sophie Job, MD, Fondation Fondamental, Créteil and Pôle de Psychiatrie et Psychologie Clinique - Centre Psychothérapique de Nancy, Laxou, France; JeanPierre Kahn, MD, PhD, Fondation Fondamental, Créteil, Pôle de Psychiatrie et Psychologie Clinique - Centre Psychothérapique de Nancy, Laxou and Université de Lorraine, Nancy, France; Marion Leboyer, MD, PhD, Fondation Fondamental, Créteil AP-HP, Hôpitaux Universitaires Henri Mondor, DHU Pepsy, Pôle de Psychiatrie et d'Addictologie, Créteil, Université Paris Est, Faculté de Médecine, Créteil and Inserm, U955, Equipe Psychiatrie Translationnelle, Créteil, France; Emilie Olié, MD, PhD, Fondation Fondamental, Créteil, Department of Emergency Psychiatry \& Post Acute Care, Academic hospital of Montpellier, Montpellier University, Montpellier and 16 INSERM U1061, Montpellier, France; Chantal Henry, MD, PhD, Fondation Fondamental, Créteil, AP-HP, Hôpitaux Universitaires Henri Mondor, DHU Pepsy, Pôle de Psychiatrie et d'Addictologie, Créteil, Université Paris Est, Faculté de Médecine, Créteil, Inserm, U955, Equipe Psychiatrie Translationnelle, Créteil and Institut Pasteur, Unité Perception et Mémoire, Paris, France; Christine Passerieux, MD, PhD, Service Unite Perception et Memoire, Paris, France; Christine Passerieux, MD, PhD, Service
Universitaire de Psychiatrie d'Adultes, Centre Hospitalier de Versailles, Le Chesnay, Laboratoire HandiRESP, EA4047, UFR des Sciences de la Santé Simone Veil, Université de Versailles Saint-Quentin-En-Yvelines, Montigny-le-Bretonneux and Fondation Fondamental, Créteil, France

Correspondence: Paul Roux, MD, PhD, Service Universitaire de Psychiatrie d'Adultes, Centre Hospitalier de Versailles, 177 rue de Versailles, 78157 Le Chesnay, France. Email: paul.roux@uvsq.fr

First received 13 May 2017, final revision 14 Jul 2017, accepted 25 Jul 2017

\section{Funding}

This work was supported by the Centre Hospitalier de Versailles, Foundation FondaMental, Créteil, France, and by the Investissements d'Avenir Programs managed by the ANR under references ANR-11-IDEX-0004-02 and ANR-10-COHO-10-01.

\section{Acknowledgements}

We thank the Centre Hospitalier de Versailles and William Hempel of Alex Edelman \& Associates for editorial assistance.

\section{Appendix}

\section{List of FACE-BD collaborators}

FACE-BD Clinical Coordinating Center (FondaMental Foundation): B. Etain, C. Henry and M. Leboyer.

FACE-BD Data Coordinating Center (FondaMental Foundation): O. Godin, H. Laouamri and N. Ngo-Nguyen.

FACE-BD clinical sites and principal collaborators in France: AP-HP, Hôpital H. Mondor - A. Chenevier, Pôle de Psychiatrie, Créteil - C. Daban, S. Hotier, S. Lauer, A. Leduc, A. Pelletier and A. Raust; AP-HP, GH SaintLouis-Lariboisière-Fernand Widal, Pôle Neurosciences, Paris - F. Bellivier, M. Carminati, B. Etain, J. P. Lépine, I Nieto, P. Seguin and S. Sportiche; 
Hôpital C. Perrens, Centre Expert Trouble Bipolaire, Service de Psychiatrie Adulte, Pôle 3-4-7, Bordeaux - B. Antoniol, A. Desage, S. Gard, A. Jutant, K. Mbailara, I. Minois and L. Zanouy; Département d'Urgence et Post Urgence Psychiatrique, CHRU Montpellier, Montpellier - S. Bonnet, F. Coppola, P. Courtet, D. Ducasse, M. Gachet, L. Matos, F. Molière, B. Noisette, E. Olié and G. Tarquini; Département de Psychiatrie, Hôpital Sainte Marguerite, Marseille - J. M. Azorin, R. Belzeaux and N. Corréard; Service de Psychiatrie et Psychologie Clinique, CHU de Nancy, Hôpitaux de Brabois, Vandoeuvre Les Nancy - R. Cohen, J.P. Kahn, P. Kieffer and O. Wajsbrot-Elgrabli; Clinique Universitaire de Psychiatrie, $\mathrm{CHU}$ de Grenoble, Grenoble - T. Bougerol, M. A. De Pourtales, B. Fredembach, S. Garçon, Alexandre Perrin and M. Polosan; Centre Hospitalier de Versailles, Service Universitaire de Psychiatrie d'adultes, Le Chesnay A.M. Galliot, I. Grévin, M. C. Hardy-Bayle, A.S. Cannavo, N. Kayser, C. Passerieux and P. Roux; Service de Psychiatrie, Centre Hospitalier Princesse Grace, Monaco - L. Albertini, V. Aubin, E. Beetz, I. Cussac, J. Loftus and I. Medecin.

\section{References}

1 Rosa AR, Franco C, Martinez-Aran A, Sanchez-Moreno J, Reinares M, Salamero $M$, et al. Functional impairment in patients with remitted bipolar disorder. Psychother Psychosom 2008; 77: 390-2.

2 Merikangas KR, Jin R, He JP, Kessler RC, Lee S, Sampson NA, et al. Prevalence and correlates of bipolar spectrum disorder in the world mental health survey initiative. Arch Gen Psychiatry 2011; 68: 241-51.

3 Sanchez-Moreno J, Martinez-Aran A, Vieta E. Treatment of functional impairment in patients with bipolar disorder. Curr Psychiatry Rep 2017; 19: 3

4 Martinez-Aran A, Vieta E, Torrent C, Sanchez-Moreno J, Goikolea JM, Salamero $\mathrm{M}$, et al. Functional outcome in bipolar disorder: the role of clinical and cognitive factors. Bipolar Disord 2007; 9: 103-13.

5 Depp CA, Mausbach BT, Harmell AL, Savla GN, Bowie CR, Harvey PD, et al. Meta-analysis of the association between cognitive abilities and everyday functioning in bipolar disorder. Bipolar Disord 2012; 14: 217-26.

6 Schretlen DJ, Pena J, Aretouli E, Orue I, Cascella NG, Pearlson GD, et al. Confirmatory factor analysis reveals a latent cognitive structure common to bipolar disorder, schizophrenia, and normal controls. Bipolar Disord 2013; 15 422-33.

7 Czobor P, Jaeger J, Berns SM, Gonzalez C, Loftus S. Neuropsychological symptom dimensions in bipolar disorder and schizophrenia. Bipolar Disord 2007; 9: 71-92.

8 Gallagher P, Gray JM, Watson S, Young AH, Ferrier IN. Neurocognitive functioning in bipolar depression: a component structure analysis. Psychol Med 2014; 44: 961-74.

9 Bonnin CM, Martinez-Aran A, Torrent C, Pacchiarotti I, Rosa AR, Franco C, et al. Clinical and neurocognitive predictors of functional outcome in bipolar euthymic patients: a long-term, follow-up study. J Affect Disord 2010; 121: 156-60.

10 Dias VV, Brissos S, Frey BN, Kapczinski F. Insight, quality of life and cognitive functioning in euthymic patients with bipolar disorder. $J$ Affect Disord 2008; 110: $75-83$.

11 Belzeaux R, Correard N, Boyer L, Etain B, Loftus J, Bellivier F, et al. Depressive residual symptoms are associated with lower adherence to medication in bipolar patients without substance use disorder: results from the FACE-BD cohort. J Affect Disord 2013; 151: 1009-15.

12 Allen DN, Bello DT, Thaler NS. Neurocognitive predictors of performancebased functional capacity in bipolar disorder. J Neuropsychol 2015; 9: 159-71.

13 Aparicio A, Santos JL, Jimenez-Lopez E, Bagney A, Rodriguez-Jimenez R, Sanchez-Morla EM. Emotion processing and psychosocial functioning in euthymic bipolar disorder. Acta Psychiatr Scand 2017; 135: 339-50.

14 Martino DJ, Marengo E, Igoa A, Scapola M, Ais ED, Perinot L, et al. Neurocognitive and symptomatic predictors of functional outcome in bipola disorders: a prospective 1 year follow-up study. J Affect Disord 2009; 116: 37-42.

15 Bourne C, Bilderbeck A, Drennan R, Atkinson L, Price J, Geddes JR, et al. Verbal learning impairment in euthymic bipolar disorder: BDI $\vee$ BDII. $J$ Affect Disord 2015; 182: 95-100.

16 Bourne C, Aydemir O, Balanza-Martinez V, Bora E, Brissos S, Cavanagh JT, et al. Neuropsychological testing of cognitive impairment in euthymic bipolar disorder: an individual patient data meta-analysis. Acta Psychiatr Scand 2013; 128: 149-62.

17 del Mar Bonnín C, González-Pinto A, Solé B, Reinares M, González-Ortega I, Alberich $\mathrm{S}$, et al. Verbal memory as a mediator in the relationship between subthreshold depressive symptoms and functional outcome in bipolar disorder. J Affect Disord 2014; 160: 50-4.

18 Bowie CR, Depp C, McGrath JA, Wolyniec P, Mausbach BT, Thornquist MH, et al. Prediction of real-world functional disability in chronic mental disorders: a comparison of schizophrenia and bipolar disorder. Am J Psychiatry 2010; 167: 1116-24.

19 American Psychiatric Association. Diagnostic and Statistical Manual of Mental Disorders (4th edn, revised) (DSM-IV-TR). APA, 2000.

20 Montgomery SA, Asberg M. A new depression scale designed to be sensitive to change. Br J Psychiatry 1979; 134: 382-9.

21 Young RC, Biggs JT, Ziegler VE, Meyer DA. A rating scale for mania: reliability, validity and sensitivity. Br J Psychiatry 1978; 133: 429-35.

22 Rosa AR, Sanchez-Moreno J, Martinez-Aran A, Salamero M, Torrent C Reinares $M$, et al. Validity and reliability of the Functioning Assessment Short Test (FAST) in bipolar disorder. Clin Pract Epidemiol Ment Health 2007; 3: 5.

23 Yatham LN, Torres IJ, Malhi GS, Frangou S, Glahn DC, Bearden CE, et al. The International Society for Bipolar Disorders-Battery for Assessment of Neurocognition (ISBD-BANC). Bipolar Disord 2010; 12: 351-63.

24 Wechsler D. Wechsler Adult Intelligence Scale (3rd edn) (WAIS-III). Psychological Corporation, 1997.

25 Conners CK, Staff M. Conners' Continuous Performance Test II. Multi-Health Systems Inc, 2000

26 Reitan RM. Validity of the Trail Making Test as an indicator of organic brain damage. Percept Mot Skills 1958; 8: 271-6.

27 Lezak MD. Neuropsychological Assessment. Oxford University Press, 2004.

28 Delis D, Kramer J, Kaplan E, Ober B. CVLT-II California Verbal Learning Test Manual Adult Version. The Psychological Corporation, 2000.

29 Roux P, Raust A, Cannavo AS, Aubin V, Aouizerate B, Azorin JM, et al. Cognitive profiles in euthymic patients with bipolar disorders: results from the FACE-BD cohort. Bipolar Disord 2017; 19: 146-53.

30 Dziuban $C D$, Shirkey EC. When is a correlation matrix appropriate for factor analysis? Some decision rules. Psychol Bull 1974; 81: 358.

31 Bartlett MS. The effect of standardization on a 2 approximation in factor analysis. Biometrika 1951; 38: 337-44.

32 Horn JL. A Rationale and test for the number of factors in factor analysis. Psychometrika 1965; 30: 179-85.

33 Bryant FB, Yarnold PR. Principal-components analysis and exploratory and confirmatory factor analysis. In Reading and Understanding Multivariate Statistics (eds In LG Grimm and PR Yarnold): 99-136). American Psychological Association, 1995.

34 Bas TO, Poyraz CA, Bas A, Poyraz BC, Tosun M. The impact of cognitive impairment, neurological soft signs and subdepressive symptoms on functional outcome in bipolar disorder. J Affect Disord 2015; 174: 336-41.

35 Bentler PM, Chou C-P. Practical issues in structural modeling. Sociol Methods Res 1987; 16: 78-117.

36 Burdick KE, Goldberg JF, Harrow M. Neurocognitive dysfunction and psychosocial outcome in patients with bipolar I disorder at 15-year follow-up. Acta Psychiatr Scand 2010; 122: 499-506.

37 Reinares M, Papachristou E, Harvey P, Mar Bonnin C, Sanchez-Moreno J, Torrent $C$, et al. Towards a clinical staging for bipolar disorder: defining patient subtypes based on functional outcome. J Affect Disord 2013; 144: 65-71.

38 Samalin L, Boyer L, Murru A, Pacchiarotti I, Reinares M, Bonnin CM, et al. Residual depressive symptoms, sleep disturbance and perceived cognitive impairment as determinants of functioning in patients with bipolar disorder. $J$ Affect Disord 2017; 210: 280-6.

39 Gorwood P, Corruble E, Falissard B, Goodwin GM. Toxic effects of depression on brain function: impairment of delayed recall and the cumulative length of depressive disorder in a large sample of depressed outpatients. Am J Psychiatry 2008; 165: 731-9.

40 Boland EM, Stange JP, Molz Adams A, LaBelle DR, Ong ML, Hamilton JL, et al. Associations between sleep disturbance, cognitive functioning and work disability in bipolar disorder. Psychiatry Res 2015; 230: 567-74.

41 Bonnin CM, Reinares M, Martinez-Aran A, Balanza-Martinez V, sole B, Torrent $C$, et al. Effects of functional remediation on neurocognitively impaired bipolar patients: enhancement of verbal memory. Psychol Med 2016; 46: 291-301. 